\title{
An Approximate Formula for the Length of an Arc of a Suspended Rope.
}

\author{
By Dr E. M. Horsburgh.
}

(Read 11th January 1918. Received s0th May 1918.

The length of an arc of a flexible rope or chain suspended in the catenary $y=c \cosh x / c$ is $s=c \sinh x / c$ when measured from thu vertex, but the practical determination of $s$ is troublesome, owing to the difficulty in finding the parameter $c$ from the transcendental equation when the coordinates of the point of suspension are given. The importance of a formula such as Huygens' approximation to the length of a circular arc $s=2 B+\frac{1}{3}(2 B-A)$, where $A$ is the chord of the arc and $B$ that of half the arc, is due to the fact that one can scale directly these lengths by rectilinear measurements without requiring to find the central angle or to make any subsidiary calculations. Formulae of this nature applicable to the parabola, or to curves whose arcs might be replaced by parabolic arcs, would be useful in the design of structural works dealing with ropes or chains. In such cases, as the dip is frequently less than one-eighth of the space, the catenary may be replaced by a parabola.

Although it is simple to write down the length of an arc of a parabola in the form of an integral, yet such a formula is troublesome to work with, and is quite unsuitable for many of the engineering problems which arise. A formula is required by means of which it is possible to read off approximately the length of an arc of the curve from a diagram by means of rectilinear measurements. In what follows certain formulae are worked out by means of which the length of the arc may be scaled or calculated with sufficient accuracy for practical purposes under certain specified conditions.

If we take the curve to be a parabola of the family $y=l x^{2}$, the length of the arc joining the points $\left(x_{1}, y_{1}\right)$ and $\left(x_{2}, y_{2}\right)$ is

$$
\begin{aligned}
& s=\int_{x_{1}}^{x_{2}} d x \sqrt{1+4 l^{2} x^{2}}=\int_{x_{1}}^{x_{2}} d x\left(1+2 l^{2} x^{2}-2 l^{4} x^{4}+\ldots\right) \\
& \quad \longrightarrow\left(x_{2}-x_{1}\right)+\frac{2}{3} l^{2}\left(x_{2}^{3}-x_{1}^{3}\right)-\frac{2}{3} l^{4}\left(x_{2}^{5}-x_{1}^{5}\right)
\end{aligned}
$$


since $l$ is small, where the symbol $\rightarrow$ denotes "is approximately equal to."

The length of the chord is $c=\sqrt{\left(x_{2}-x_{1}\right)^{2}+l^{2}\left(x_{2}^{2}-x_{1}^{2}\right)^{2}}$

$$
\longrightarrow\left(x_{2}-x_{1}\right)\left\{1+\frac{1}{2} l^{2}\left(x_{1}+x_{1}\right)^{2}-\frac{1}{8} l^{4}\left(x_{2}+x_{1}\right)^{4}\right\} \text {. }
$$

Let the vertical sagitta of the middle point of the chord be denoted by $\sigma$. The abscissa of the middle point is $\frac{1}{2}\left(x_{1}+x_{2}\right)$. Let $\eta_{1}$ be the ordinate of the curve at this point, and $\eta_{2}$ that of the chord.

$$
\begin{aligned}
& \therefore \quad \eta_{1}=\frac{l}{4}\left(x_{1}+x_{2}\right)^{2} \quad \eta_{2}=\frac{1}{2} l\left(x_{1}^{2}+x_{2}^{2}\right) . \\
& \therefore \quad \eta_{2}-\eta_{1}=\sigma=\frac{l}{4}\left(x_{2}-x_{1}\right)^{2} . \\
& \therefore \quad \operatorname{arc}-\operatorname{chord}=\frac{l^{2}}{6}\left(x_{2}-x_{1}\right)^{3}+\ldots \\
& \quad \operatorname{arc} \rightarrow \text { chord }+\frac{8}{3} \frac{\sigma^{2}}{\left(x_{2}-x_{1}\right)} \ldots \ldots . .
\end{aligned}
$$

Hence

If this value of $\sigma$ be expressed in terms of the coordinates of the extremities of the arc, we have

$$
\operatorname{arc} \rightarrow \text { chord }+\frac{1}{6} \frac{\left(x_{2}-x_{1}\right)\left(y_{2}-y_{1}\right)^{2}}{\left(x_{2}+x_{1}\right)^{2}}
$$

The length of the arc may also be expressed in terms of the chord and tangents at its extremities. The equations of the tangents at the points $\left(x_{1}, y_{1}\right)$ and $\left(x_{2}, y_{2}\right)$ are $y+y_{1}=2 l x x_{1}$ and $y+y_{2}=2 l x x_{2}$, and their point of intersection is $\left\{\frac{1}{2}\left(x_{1}+x_{2}\right), l x_{1} x_{2}\right\}$.

Let $t_{1}$ and $t_{2}$ be the lengths of these tangents. Therefore $t_{1}=\left(x_{2}-x_{1}\right)\left\{\frac{1}{4}+l^{2} x_{1}^{2}\right\}^{\frac{1}{2}}$, with a corresponding expression for $t_{2}$.

Hence $\quad T=t_{1}+t_{2} \rightrightarrows\left(x_{2}-x_{1}\right)\left\{1+l^{2}\left(x_{1}^{2}+x_{2}^{2}\right)\right\}$.

Eliminating $x_{1}$ and $x_{2}$ between these three equations, which are all approximately true for $T, C$, and $s$, we have an expression for the length of the arc

$$
s \geq \frac{2}{3} C+\frac{1}{3} T,
$$

where $C$ is the length of the chord of the arc and $T$ the sum of the lengths of the tangents. The tangents are easy to draw accurately from the well-known properties of double tangents to a parabola, so the length of the arc is simple to determine. For a dip of $100^{\prime}$ and a span of $600^{\prime}$, the last formula gives a calculated error of only about $.008 \%$, so that the error caused in examples such as this is negligible from the graphical point of view. 